\title{
Expression of HSP90AAI/HSPA8 in hepatocellular carcinoma patients with depression
}

This article was published in the following Dove Press journal:

OncoTargets and Therapy

\author{
Xiao Xiang' \\ Xue-Mei You ${ }^{1,2}$ \\ Le-Qun $\mathrm{Li}^{1,2}$ \\ 'Department of Hepatobiliary \\ Surgery, Affiliated Tumor Hospital \\ of Guangxi Medical University, \\ Nanning, People's Republic of China; \\ ${ }^{2}$ Guangxi Liver Cancer Diagnosis and \\ Treatment Engineering and Technology \\ Research Center, Nanning, People's \\ Republic of China
}

Background: Depression may influence susceptibility to cancer, and the genes and signaling pathways that may mediate this association are unclear.

Methods: Here, we used isobaric tagging for relative and absolute quantitation, 2-dimensional liquid chromatography, and mass spectrometry to compare proteins expressed in hepatocellular carcinoma in patients with or without depression.

Results: A total of 89 proteins were up-regulated and 44 were down-regulated in patients with depression. HSP90AA1 and HSPA8 were up-regulated, which correlated with elevated levels of VEGF, VEGFR2, PI3K, and AKT1 and reduced levels of caspase 9 and BAD. Disease-free survival rate was significantly lower and risk of tumor recurrence was significantly higher in patients with depression, which may reflect high HSP90AA1/HSPA8 expression.

Conclusion: These results suggest that the VEGF/VEGFR2 pathway may be associated with HCC recurrence in patients expressing high levels of HSP90AA1/HSPA8.

Keywords: heat shock protein 90AA1, heat shock protein A8, depression, hepatocellular carcinoma, VEGF

\section{Introduction}

Hepatocellular carcinoma (HCC) is one of the most common malignancies in the world. ${ }^{1}$ An average of 700,000 new cases are diagnosed each year, 50\% of which occur in People's Republic of China. ${ }^{2}$ The Guangxi area has a high incidence of liver cancer, where it is associated with a crude mortality rate of 27.31 per 10 million (41.78 males and 11.71 females per 10 million), placing it first among all types of malignant tumors. ${ }^{3}$ Some HCC patients may chronically experience negative emotions related to their health condition, especially because of the lack of appropriate long-term psychological counseling and social support.

This negative emotional state, like other psychosocial factors, may reduce the number of immune cells and cytokine secretion ${ }^{4}$ by influencing the axis comprising the hypothalamic-pituitary-adrenal gland, gonads, and thyroid, which regulates neurotransmitter secretion. ${ }^{5}$ In this way, negative emotional state may increase susceptibility to cancer and other diseases. ${ }^{6}$ In fact, at least one study has suggested that cancer susceptibility may be higher among individuals who constantly struggle to meet the needs of others, compromise their own desires for the sake of others, and suppress negative emotions. ${ }^{7}$ Incidence of cancer may be more than threefold greater among individuals with depression than among others, ${ }^{8}$ and one study found depression to be present among $74.1 \%$ of cancer patients. ${ }^{9}$ Depression may increase cancer risk by weakening the body's ability to conduct immune surveillance and repair damaged DNA. This facilitates the transformation of proto-oncogenes, inducing tumor growth, recurrence, and metastasis. 
Liver cancer patients show varying extents of depression, anxiety, and other adverse emotions. Moderate psychological intervention can significantly improve the quality of life of patients with liver cancer. ${ }^{10,11}$ If biomarkers of depressionrelated liver cancer could be identified, they might enable earlier diagnosis and more personalized prevention and treatment.

In order to identify such biomarkers and help elucidate HCC pathways that may occur in a background of depression, we performed isobaric tagging of proteins in tumor tissues from HCC patients with or without depression. We identified 89 proteins that were upregulated and 44 that were downregulated in the presence of depression. Gene ontology and pathway analyses were performed, and protein-protein interaction (PPI) networks and modular analyses were carried out to identify central (hub) proteins in depression-related HCC. Potential correlations of these hub proteins and key pathways with clinicopathological features and prognosis were explored.

\section{Methods}

The study protocol for this trial was approved by the Guangxi Medical University Affiliated Tumor Hospital Ethics Committee and was designed in accordance with the Helsinki Declaration (2013 version). Written informed consent was obtained from all patients.

\section{Patients and tissue samples}

Fresh HCC tissues were taken from the Tumor Tissue Bank of the Affiliated Tumor Hospital of Guangxi Medical University. Tissues had been removed from 131 patients with HCC who underwent liver resection at the Affiliated Tumor Hospital of Guangxi Medical University between June 2014 and February 2015.

\section{Diagnostic criteria}

HCC tissues were taken from patients who had been diagnosed with HCC strictly according to the guidelines of the American Association for the Study of Liver Diseases. ${ }^{12}$ Tumors were staged according to the Barcelona Clinic Liver Cancer system. ${ }^{13}$

\section{Inclusion criteria}

HCC tissues were included if the patient 1) had been diagnosed with HCC based on liver tissue pathology; 2) could understand and communicate well enough to complete the depression assessments in this study; 3) had no personal or family history of mental illness or unconsciousness; and 4) provided written informed consent to participate in the study.

\section{Exclusion criteria}

Patients and their tumor tissue were excluded from the study if they 1) were diagnosed with a condition other than HCC; 2 ) were diagnosed with a combination of HCC and another malignancy; 3) could not understand or communicate sufficiently to complete the depression assessments in this study; 4) had personal or family history of mental illness; or 5) did not want to participate in this study.

\section{Follow-up}

All patients were followed-up at 1 month after hepatectomy and every 3 months thereafter. During each follow-up visit, the following tests were performed: chest X-ray, abdominal computed tomography or magnetic resonance imaging, serum alpha-fetoprotein assay, and abdominal ultrasonography. The last follow-up was August 2017.

\section{Depression assessment}

All patients in the study were assessed for the presence and severity of depression. The Self-Rating Depression Scale $(\mathrm{SDS})^{14}$ features 20 items that are scored to yield a depression severity index (total score/80). An index below 0.50 is considered to indicate no depression; $0.50-0.59$, mild depression; 0.60-0.69, moderate depression; and $>0.70$, severe depression. The Hospital Anxiety and Depression Scale-Depression $(\text { HADS-D })^{15}$ has 14 items, each of which is assigned $0-3$ points. Overall scores of $0-7$ are considered to indicate no depression; 8-10, suspected depression; and 11-21, likely depression. For the purposes of the present study, we defined no depression as SDS $<0.5$ and HADS-D $<7$; mild depression, $0.5<\mathrm{SDS}<0.59$ and $8<\mathrm{HADS}-\mathrm{D}<10$; and moderate/ severe depression, $\mathrm{SDS}>0.6$ and HADS-D $>11$.

\section{Proteomic analysis}

Total protein was extracted from tumor tissues, and protein concentration was determined using the Bradford method and confirmed by sodium dodecyl sulfate-polyacrylamide gel electrophoresis. Proteins were reduced, cysteines were blocked, and proteins were digested with trypsin (trypsin: protein, 1:40). The trypsin was added, the mixture was vortexed, and the digestion was allowed to proceed overnight at $37^{\circ} \mathrm{C}$. The differential marker for isobaric tagging for relative and absolute quantitation (iTRAQ) was separated by reverse-phase liquid chromatography on a reversephase separation column (ZORBAX 300SB-C18 column, $5 \mu \mathrm{m}, 300 \AA, 0.1-15 \mathrm{~mm}$ [Microm, Auburn, CA, USA]). 
Eluted polypeptides were analyzed using tandem mass spectrometry on an online QSTAR Pulsar ${ }^{\mathrm{TM}}$ XL MS/MS system (Applied Biosystems-MDS Sciex, Foster City, CA, USA) and RPLC column (ZORBAX 300SB-C18 column, 5 Hm, $300 \AA$, 0.1-15 mm [Microm]). The iTRAQ-tagged peptides were fragmented at $113.1,116.1,117.1,118.1$, and 119.1 on CID, generating reporter ions while also producing peptide fragment ions. This allowed sequencing of the tagged peptides and identification of the corresponding proteins. The ratio of peak areas for the three iTRAQ reporter ions reflects the relative abundance of peptides and proteins in the sample.

\section{Gene ontology and pathway enrichment analysis}

Candidate functions and pathways enriched in the presence of depression were analyzed using several online databases, including DAVID, KEGG PATHWAY (www.genome.jp/ kegg), Reactome (www.reactome.org), BioCyc (biocyc.org), and Funrich V3 software. Ontology analysis of differentially expressed proteins was performed using DAVID and Funrich version 3 . In most analyses, the definition of statistical significance was $P<0.05$.

\section{PPI network and modular analysis}

PPI network was analyzed using the STRING website (http:// string-db.org). Potential relationships between candidate differentially expressed proteins and the degree of nodes were identified using Cytotype software. Proteins in central nodes may be hub proteins with important regulatory functions. The most significant modules from the PPI network were analyzed further using Cytotype MCODE.

\section{Quantitative RT-PCR}

Total RNA was extracted from tumor tissue using Trizol reagent (Thermo Fisher Scientific, Waltham, MA, USA). Complementary DNA was generated using the PrimeScript reverse transcription kit (Takara, Tokyo, Japan) according to the manufacturer's instructions. Primers were designed according to the Genbank database (National Center for Biotechnology Information, Bethesda, MD, USA) and Primer 6.0 software (Primer-E, Auckland, New Zealand). Results were analyzed using the ABI StepOne Plus System (Applied Biosystems) Levels of target mRNAs were quantitated relative to levels of glyceraldehyde3-phosphate dehydrogenase mRNA using the $\Delta C_{t}$ method.

\section{Immunohistochemistry}

Tissue sections were embedded in paraffin, dewaxed, hydrated, and subjected to antigen recovery with pressurized cooking. Samples were incubated with rabbit monoclonal anti-HSPA8/90AA1 antibody (1:1,000; Abcam, Cambridge, UK), washed, and incubated with goat anti-rabbit biotinconjugated secondary antibody (ZSGB, Beijing, People's Republic of China), then stained with 3,3-diaminobenzidine tetrahydrochloride. HSPA8/90AA1 protein expression was quantitated based on integrated optical density of antibody staining.

\section{Western blotting}

HCC tissues were lysed in RIPA (Solarbio, Beijing, People's Republic of China) buffer containing $1 \mathrm{mmol} / \mathrm{L}$ phenylmethanesulfonyl fluoride (Solarbio), and proteins were collected by centrifugation at $12,000 \times g$ for 10 minutes at $4^{\circ} \mathrm{C}$. Protein concentration was determined using a bicinchoninic acid kit (Beyotime, Shanghai, People's Republic of China). Membranes were blocked for 2 hours with 5\% skim milk in PBS containing Tween 20, and then incubated with rabbit anti-HSPA8/HSP90AA1 mAb (1:1,000, Abcam) overnight at $4^{\circ} \mathrm{C}$. After washing 3 times each for 5 minutes in PBS containing Tween 20, membranes were incubated with goat anti-rabbit horseradish peroxidase-conjugated antibody $(1: 5,000)$ for 2 hours at room temperature. Bands were observed using enhanced chemiluminescence (Beyotime). All experiments were performed 3 times.

\section{Statistical analysis}

Data were reported as mean $\pm \mathrm{SD}$ or median and range as appropriate. Intergroup differences in categorical data were assessed for significance using the $\chi^{2}$ test or Fisher's exact test (2-tailed), whereas differences in continuous data were assessed using the Mann-Whitney $U$ test. Overall survival was analyzed using the Kaplan-Meier method and compared between groups using the log-rank test. Multivariate Cox proportional hazard modeling was performed to identify independent prognostic factors based on adjusted HRs and associated 95\% CIs. All statistical analyses were performed using SPSS 20.0 (IBM Corporation, Armonk, NY, USA). For all tests, $P<0.05$ was considered statistically significant.

\section{Results}

\section{Identification of proteins differentially expressed in depression-related HCC}

Twenty patients were assessed for the presence and severity of depression based on the HADS-D and SDS scales. In the end, 10 patients were assigned to a group with moderate/severe 
depression, whereas the other 10 were assigned to a control group with no or mild depression. The combination of iTRAQ, 2-dimensional liquid chromatography and tandem mass spectrometry identified 133 proteins differentially expressed between the 2 patient groups: 89 proteins were upregulated and 44 were downregulated in the presence of moderate/severe depression (Figure 1A, Table S1).

\section{Ontology analysis of differentially expressed proteins}

Proteins differentially expressed in the presence of moderate/ severe depression could be divided into a molecular functional group, biological process group, and cell component group (Figure 1C and D, Tables S2 and $\underline{\mathrm{S} 3}$ ).
Analysis of signaling pathway enrichment

Proteins upregulated in the presence of moderate/severe depression comprised proteins involved in signaling events mediated by proteoglycan syndecan-1, VEGF, VEGFR1, VEGFR2, and $\alpha 9 \beta 1$ integrin. Proteins downregulated in the presence of moderate/severe depression are involved mainly in biological oxidations, D-glucuronate degradation I, platelet degranulation, fat metabolism, and glucose metabolism (Figure 1E, Tables S2 and S3).

\section{PPI analysis}

The PPI network contained 101 nodes and 575 edges. Applying a filter of degree $>10$ criteria, we identified 10 central node proteins: HSP90AA1, HSP90AB1, HSPA8, PIK3CA,
A

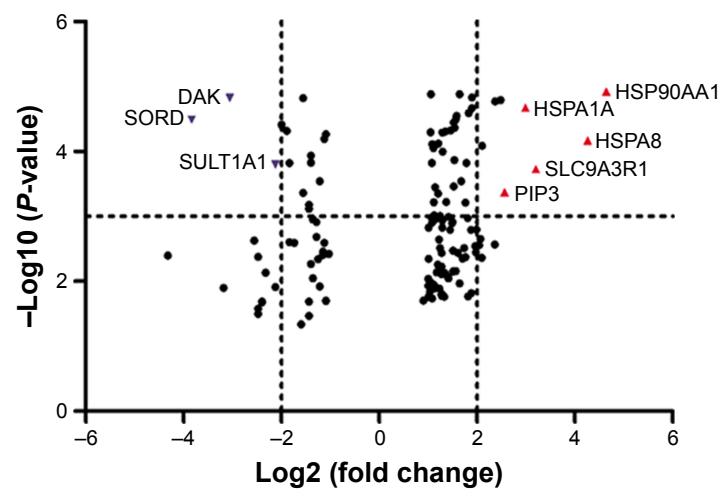

B

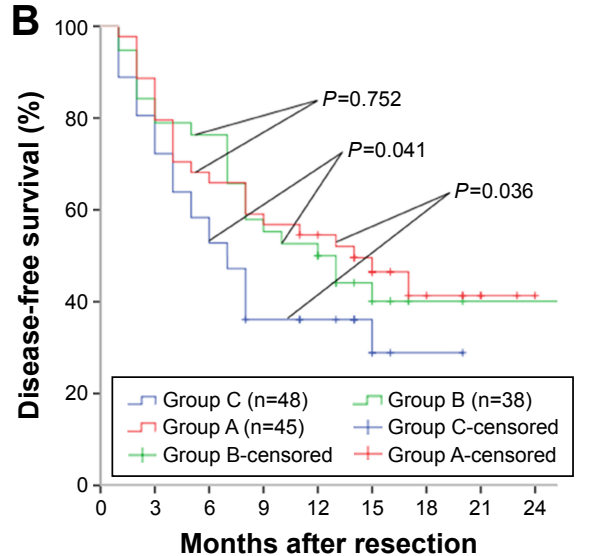

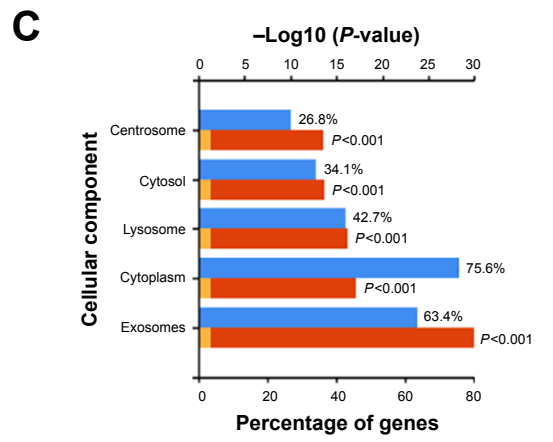

D

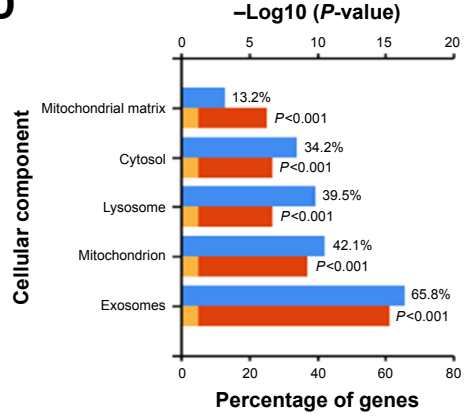

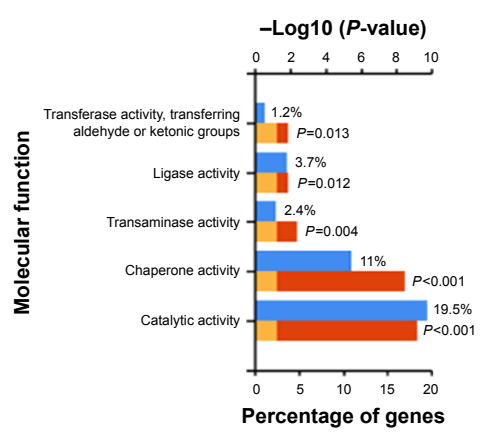
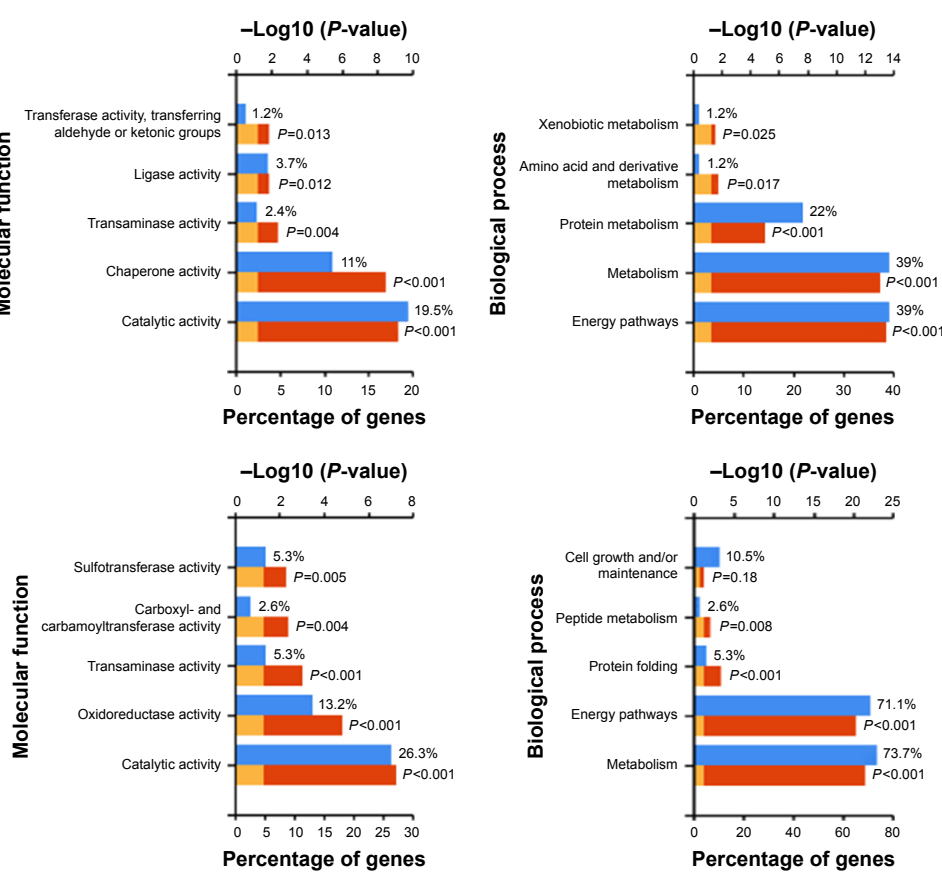

Percentage of genes

Percentage of gene $P=0.05$ reference $P$-value

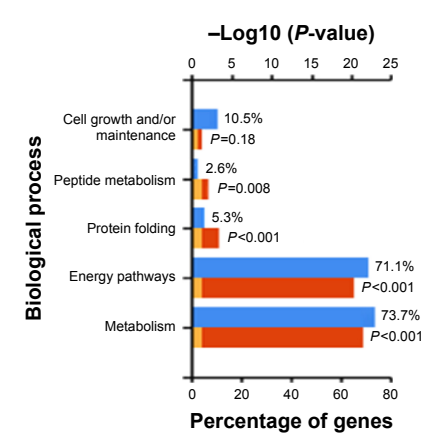

Figure I (Continued) 

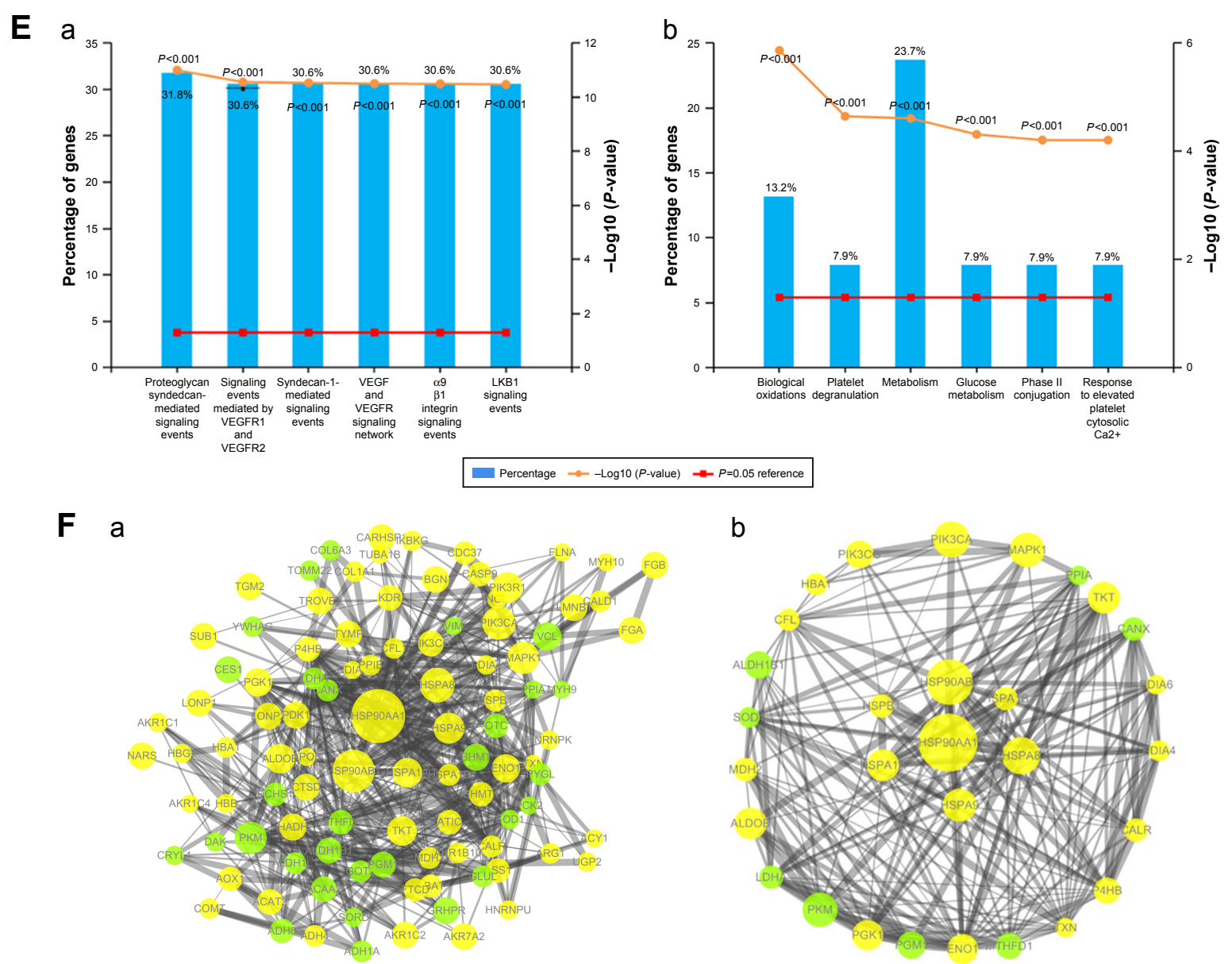

Figure I Volcanic plot analyses of iTRAQ data showing proteins differentially expressed with fold changes $>2.0(P<0.05)$ between patients with moderate/severe or mild/ no depression (A). DFS curves for patients with hepatocellular carcinoma involving varying degrees of depression (B). Gene ontology analysis (molecular function, biological process, and cellular component) of proteins upregulated (C) or downregulated (D) in the presence of moderate/severe depression. Functional analysis of differentially expressed proteins and signaling pathway enrichment were conducted using online websites of the KEGG PATHWAY of proteins upregulated (Ea) or downregulated (Eb). PPI network and module analysis of differentially expressed proteins (Fa). Module analysis showing 30 nodes and 206 edges (Fb).

Note: Group A: no depression, group B: mild depression, group C: moderate/severe depression.

Abbreviations: DFS, disease-free survival; PPI, protein-protein interaction.

PKM, MAPK1, HSPA9, TKT, HSPA1A, and ALDOB. Applying a filter of betweenness $>1,000$, we identified 8 central node proteins: HSP90AA1, HSPA8, ALDH1B1, CANX, ENO1, HSP90AB1, PGM1, and P4HB. In this way, HSP90AA1 and HSPA8 emerged as significant hub proteins (Figure 1Fa). We chose the most significant modules from the PPI network for further analysis by Cytotype MCODE (Figure $1 \mathrm{Fb}$ ).

These results suggest that HSP90AA1 and HSPA8 may be important hub proteins in depression-related HCC and may affect prognosis via the VEGF/VEGFR2-PI3K-AKT signaling pathway (Figures 1 and $\underline{\mathrm{S} 1}$ ).

\section{Study population}

A total of 131 patients (112 males) with a median age of 49 years were enrolled in this prospective study, of whom
45 showed no depression, 38 showed mild depression, and 48 showed moderate/severe depression based on the HADS-D and SDS scales. Clinicopathological characteristics are presented in Table 1. The 3 patient groups did not differ significantly (all $P>0.05$ ) except that patients with moderate/severe depression had significantly larger tumors and higher aspartate transaminase levels than the other 2 groups (all $P<0.05$ ).

\section{Depression and other predictors of poor disease-free survival (DFS)}

A total of 67 (51.1\%) patients experienced tumor recurrence within 18 months after liver resection, including 20 (44.4\%) with no depression, 18 (47.3\%) with mild depression, and 29 (60.4\%) with moderate/severe depression. Median DFS was 10 months among all patients, 8 months among patients with moderate/severe depression, 14 months among patients 
Table I Baseline characteristics of I3I patients with HCC who underwent liver resection

\begin{tabular}{|c|c|c|c|c|}
\hline Variable & $\begin{array}{l}\text { Group A } \\
(n=45)\end{array}$ & $\begin{array}{l}\text { Group B } \\
(n=38)\end{array}$ & $\begin{array}{l}\text { Group C } \\
(n=48)\end{array}$ & $P$-value \\
\hline Gender & & & & 0.262 \\
\hline Male & 34 & 34 & 39 & \\
\hline Female & 11 & 4 & 9 & \\
\hline Age, years & $49.5 \pm 10.7$ & $49.0 \pm 11.1$ & $47.9 \pm 10.2$ & 0.110 \\
\hline Tumor size, cm & $7.9 \pm 3.3$ & $8.5 \pm 4.3$ & $8.1 \pm 4.8$ & 0.057 \\
\hline Tumor number & & & & 0.847 \\
\hline Single & 29 & 26 & 30 & \\
\hline Multiple & 16 & 12 & 18 & \\
\hline Cirrhosis & & & & 0.774 \\
\hline Absent & 3 & 4 & 5 & \\
\hline Present & 42 & 34 & 43 & \\
\hline $\mathrm{HBsAg}, \pm$ & $31 / 14$ & $27 / 11$ & $33 / 15$ & 0.969 \\
\hline $\mathrm{HCsAg}, \pm$ & $\mathrm{I} / 44$ & $0 / 38$ & $1 / 47$ & 0.659 \\
\hline AFP, $\mathrm{ng} / \mathrm{mL}$ & & & & 0.292 \\
\hline$\geq 400$ & 19 & 20 & 28 & \\
\hline$<400$ & 26 & 18 & 20 & \\
\hline Macrovascular invasion & & & & $0.94 I$ \\
\hline Yes & 7 & 7 & 8 & \\
\hline No & 38 & 31 & 40 & \\
\hline $\mathrm{BCLC}$ stage & & & & 0.807 \\
\hline$A$ & 23 & 23 & 27 & \\
\hline B & 16 & 9 & 13 & \\
\hline C & 6 & 6 & 8 & \\
\hline ALB, g/dL & $42.5 \pm 5.1$ & $43.0 \pm 3.9$ & $40.8 \pm 4.1$ & 0.055 \\
\hline PT, sec & $13.4 \pm 1.3$ & $13.4 \pm 1.4$ & $13.7 \pm 1.1$ & 0.671 \\
\hline ALT, IU/L & $42.0 \pm 28.0$ & $45.4 \pm 27.0$ & $49.6 \pm 24.6$ & 0.311 \\
\hline AST, IU/L & $45.4 \pm 26.8$ & $48.0 \pm 25.4$ & $62.2 \pm 51.3$ & $<0.001$ \\
\hline Direct bilirubin, g/dL & $5.8 \pm 2.0$ & $5.6 \pm 2.8$ & $5.7 \pm 3.2$ & 0.233 \\
\hline Total bilirubin, mg/dL & $11.6 \pm 4.9$ & $12.3 \pm 5.7$ & $13.3 \pm 5.4$ & 0.174 \\
\hline Platelet count, $\mu / L$ & $197.3 \pm 82.3$ & $197.9 \pm 85.7$ & $200.1 \pm 87.5$ & 0.138 \\
\hline SDS score & $0.23 \pm 0.11$ & $0.54 \pm 0.05$ & $0.68 \pm 0.17$ & $<0.001$ \\
\hline HADS score & $3.17 \pm 2.67$ & $8.25 \pm 1.71$ & $16.47 \pm 2.89$ & $<0.001$ \\
\hline
\end{tabular}

Notes: Values are $\mathrm{n}$ or $\mathrm{n}(\%)$ or mean \pm SD. Group A: no depression, group B: mild depression, group C: moderate/severe depression.

Abbreviations: AFP, alpha-fetoprotein; ALB, albumin; ALT, alanine aminotransferase; BCLC, Barcelona Clinic Liver Cancer; HADS, Hospital Anxiety and Depression Scale; HCC, hepatocellular carcinoma; PT, prothrombin time; SDS, Self-Rating Depression Scale.

with no depression, and 13 months among patients with mild depression. DFS was similar between patients with no or mild depression $(P=0.810)$.

DFS rates among all patients were $65.3 \%$ at 6 months, $47.3 \%$ at 12 months, and $36.7 \%$ at 18 months. DFS rate was significantly lower in patients with moderate/severe depression than in patients with no and mild depression at 6 months (52.8\% vs $64.8 \% / 65.9 \%), 12$ months $(36.1 \%$ vs $50.0 \% / 52.1 \%)$, and 24 months $(28.9 \%$ vs $40.1 \% / 41.6 \%)$ (Figure 1B). Uni- and multivariate analyses identified macrovascular invasion, high HSP70 expression, and moderate/ severe depression as predictors of poor DFS (Table 2).

\section{Expression of HSP90AAI and HSPA8 in HCC tissues}

Expression was assessed at the mRNA level by quantitative reverse transcription polymerase chain reaction (qRT-PCR) and at the protein level by Western blotting and immunohistochemistry. Mean HSPA8 mRNA level was significantly higher in patients with moderate/severe depression $(\mathrm{n}=45)$ than in patients with no depression $(\mathrm{n}=48, P<0.001)$ or mild depression $(\mathrm{n}=38$, $P<0.001$; Figure 2A). Similarly, mean HSP90AA1 mRNA level was significantly higher in patients with moderate/severe depression $(\mathrm{n}=45)$ than in patients with no depression $(\mathrm{n}=48$, $P<0.01)$ or mild depression ( $\mathrm{n}=38, P<0.001$; Figure $2 \mathrm{~A}$ ). There was no significant difference in HSP90AA1 mRNA level between patients with no or mild depression $(P=0.891)$.

Consistent with these results at the mRNA level, Western blotting showed significantly higher levels of HSP90AA1 and HSPA 8 proteins in patients with moderate/severe depression than in the other 2 groups (Figures $2 \mathrm{~B}$ and $\underline{\mathrm{S} 2}$ ). Similarly, immunohistochemistry indicated higher levels of HSP90AA1 and HSPA8 in the presence of moderate/ severe depression (Figure 2C). These findings suggest that HSPA8 and HSP90AA1 may be biomarkers of HCC-related to moderate/severe depression.

\section{Correlation of HSP90AAI/HSPA8 upregulation with upregulation of proteins in the VEGF/VEGFR2-PI3K-AKT pathway}

Western blotting and qRT-PCR showed higher levels of HSP90AA1, HSPA8, VEGF, VEGFR2, PI3K, and AKT1 expression in patients with moderate/severe depression than in the other patient groups as well as lower caspase 9 and BAD expression (Figure 3).

\section{Discussion}

Our results with a population from Guangxi, which has a high incidence of liver cancer, ${ }^{16-18}$ suggest that a substantial proportion of patients suffer depression and that this may affect prognosis. Posthepatectomy DFS in our cohort was significantly longer, and DFS rates higher, among patients with no or mild depression than among those with moderate/ severe depression, and poor DFS correlated with higher HSP90AA1/HSPA8 expression. Our comprehensive analysis of proteins differentially expressed between HCC patients with moderate/severe or mild/no depression indicates a correlation between high HSP90AA1/HSPA8 expression and activation of the VEGF/VEGFR2-PI3K-AKT pathway. It is tempting to speculate that this activation contributes to poor 
Table 2 Uni- and multivariate analyses to identify factors influencing early tumor recurrence after liver resection in patients with HCC

\begin{tabular}{|c|c|c|c|c|c|c|}
\hline \multirow[t]{2}{*}{ Variable } & \multicolumn{3}{|c|}{ Univariate } & \multicolumn{3}{|c|}{ Multivariate } \\
\hline & HR & $95 \% \mathrm{Cl}$ & $P$-value & HR & $95 \% \mathrm{Cl}$ & $P$-value \\
\hline Age $>60$ years & 1.473 & $0.836-2.595$ & 0.180 & & & \\
\hline Male gender & 1.367 & $0.689-3.174$ & 0.466 & & & \\
\hline Tumor $>10 \mathrm{~cm}$ & 0.931 & $0.554-1.564$ & 0.787 & & & \\
\hline Multiple tumors & 0.815 & $0.482-1.378$ & 0.444 & & & \\
\hline AST $>40 \mathrm{U} / \mathrm{L}$ & $0.7 \mid 4$ & $0.439-1.662$ & 0.175 & & & \\
\hline Serum AFP $>400 \mathrm{ng} / \mathrm{mL}$ & 1.289 & $0.790-2.102$ & 0.309 & & & \\
\hline $\mathrm{ALT}>40 \mathrm{U} / \mathrm{L}$ & 1.166 & $0.719-1.892$ & 0.533 & & & \\
\hline Total bilirubin $>12 \mathrm{mg} / \mathrm{dL}$ & 0.957 & $0.590-1.553$ & 0.860 & & & \\
\hline Direct bilirubin $>7 \mathrm{~g} / \mathrm{dL}$ & 1.348 & $0.783-2.320$ & $0.28 \mathrm{I}$ & & & \\
\hline ALB $>40 \mathrm{~g} / \mathrm{dL}$ & 1.147 & $0.667-1.973$ & 0.619 & & & \\
\hline Platelets $>100 \mu / \mathrm{L}$ & 0.724 & $0.358-1.465$ & 0.369 & & & \\
\hline PT $>13 \mathrm{sec}$ & 1.102 & $0.660-1.839$ & 0.710 & & & \\
\hline Macrovascular invasion & $\mathrm{I} .457$ & $1.127-3.021$ & 0.038 & 1.476 & I.II4-2.96| & 0.040 \\
\hline BCLC stage $C$ & $\mathrm{I} .064$ & $0.7 \mid I-1.468$ & 0.705 & & & \\
\hline High HSP70 expression & 2.191 & $1.172-4.312$ & 0.017 & 2.312 & $\mathrm{I} .40 \mathrm{I}-4.94 \mathrm{I}$ & 0.011 \\
\hline Moderate/severe depression & 2.380 & $|.342-4.34|$ & $<0.001$ & 2.527 & I.457-5.34। & $<0.001$ \\
\hline
\end{tabular}

Abbreviations: AFP, alpha-fetoprotein; ALB, albumin; ALT, alanine aminotransferase; AST, aspartate transaminase; BCLC, Barcelona Clinic Liver Cancer; HCC, hepatocellular carcinoma; PT, prothrombin time.

DFS by inducing endothelial cell proliferation and migration, which promotes angiogenesis and tumor growth, ${ }^{19}$ as well as by inhibiting expression of BAD and caspase 9, which reduces tumor cell apoptosis. ${ }^{20}$ In this way, the present study has generated testable hypotheses for future research that may improve our understanding of depression-related HCC and identify biomarkers that can detect it early.

HSP90AA1 is a chaperone that is highly conserved among eukaryotes. It plays an important role in tumor cell proliferation, differentiation, survival, and movement as well as angiogenesis. It is an emerging target for tumor therapy. ${ }^{21}$ HSP90AA1 is highly expressed in a variety of malignancies including breast, endometrial, ovarian, colon, lung, and prostate cancers. ${ }^{22-24}$ During malignant tumor growth, it helps regulate mitochondrial apoptosis and signaling transduction triggered by the death receptor, stress signals, and growth factors. ${ }^{25}$ HSP90AA1 can inhibit the initiation of apoptosis by preventing the binding of caspase 9 to apoptotic protein 1 activator. ${ }^{20}$ In human leukemia cells, HSP90AA1 is inhibited and tyrosine kinase, Akt protein kinase B, and serinethreonine kinase are degraded, so that cells avoid apoptosis and undergo differentiation. ${ }^{26}$ HSP90AA1 also promotes tumor formation by stabilizing mutant p53 complexes and thereby inhibiting apoptosis. ${ }^{27}$ The ATPase inhibitor kaloxin inhibited proteolytic degradation of caspase 9 by activity of HSP90AA $1 .{ }^{28}$ In NIH3T3 cells, HSP90AA1 overexpression can inhibit apoptosis induced by tumor necrosis factor- $\alpha .{ }^{29}$

HSPA8 are stress proteins, and their overexpression in depression-related HCC may reflect that depression can expose patients to chronic stress. HSPA8 is involved in the regulation of tumor cell proliferation and apoptosis, which is closely related to the development, biological behavior, and prognosis of $\mathrm{HCC}$ and other tumors. ${ }^{32}$ In early liver cancer, HSPA8 is expressed abnormally in the cytoplasm and nucleus, and its expression increases with disease progression, whereas its expression remains negligible or low in precancerous lesions and proliferative nodules. So, HSPA8 detection can be used for early detection of liver cancersensitive indicators. In fact, HSPA8 has been suggested as an early biomarker of liver cancer. ${ }^{33}$ The upregulation of HSPA8 in depression-related HCC may be an anticancer response. Udono and Srivastava ${ }^{30}$ have shown that HSPA8 in tumor cells binds to tumor-specific antigenic polypeptides to facilitate their recognition by the host immune system. HSPA8 can also induce maturation of antigen-presenting cells, promoting the transformation of Th cells into Th1 cells, directly activating TCR $\gamma \delta \mathrm{T}$ cells and natural killer cells. ${ }^{31}$

Activation of the signal transduction pathway of VEGF and its receptor VFGFR can stimulate the proliferation and migration of vascular endothelial cells, angiogenesis in HCC, and promote tumor growth and metastasis. ${ }^{34} \mathrm{HCC}$ cells must form new blood vessels to obtain sufficient oxygen and nutrition to support their rapid growth. ${ }^{35}$ This rapid growth can lead to hypoxia within tumors, triggering expression of hypoxia-inducible factor and VEGF. VEGF production is also induced by matrix metalloproteinases, IFN- $\alpha$ and $-\gamma$, and NF-KB during HCC progression. ${ }^{36}$ VEGF binds to its receptor VEGFR to activate a series of signal transduction pathways, 

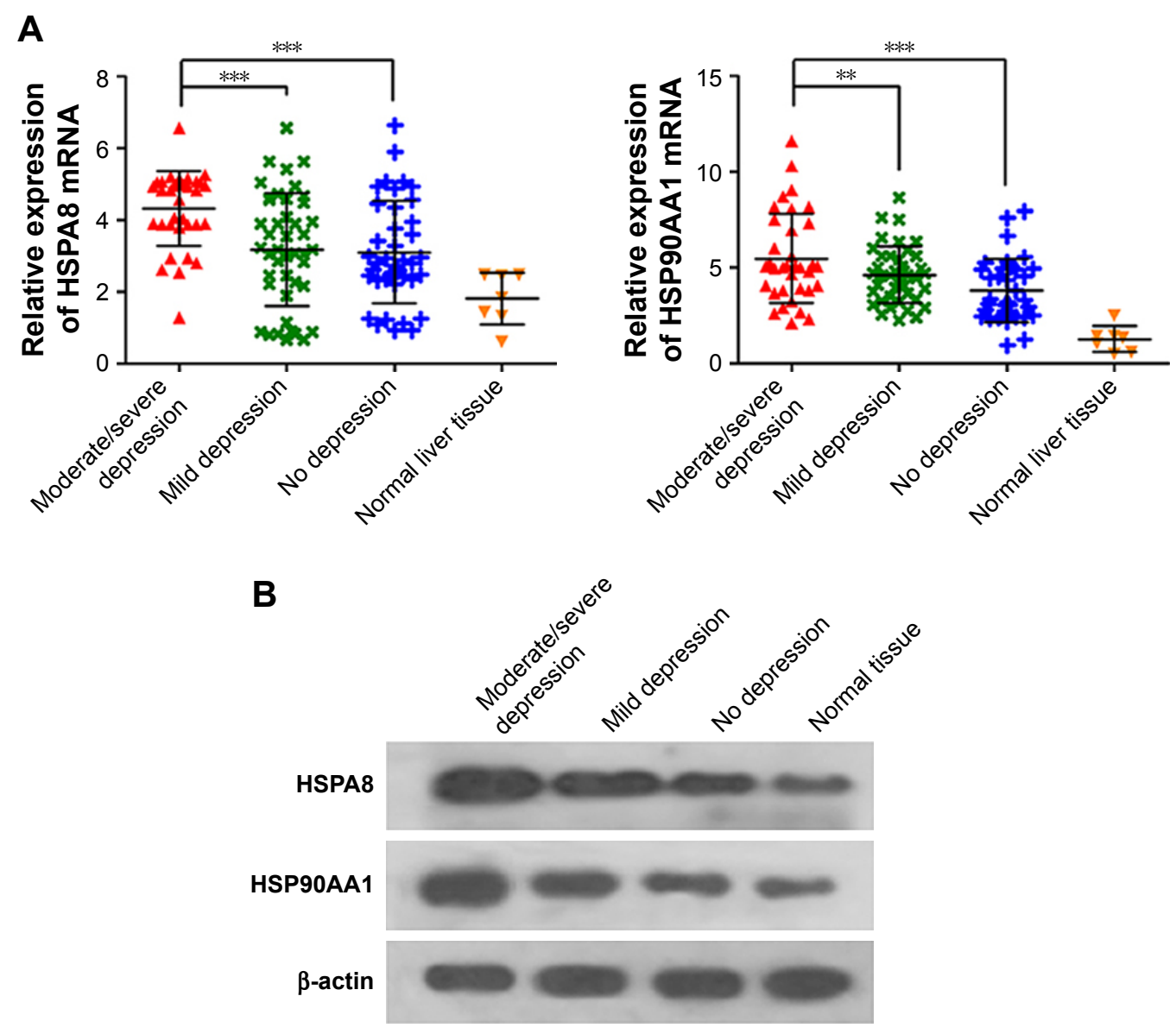

\section{Moderate/severe}
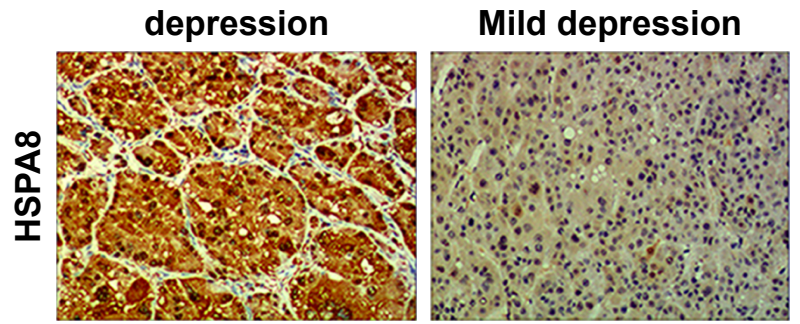

IOD: $228.1 \pm 15.2$

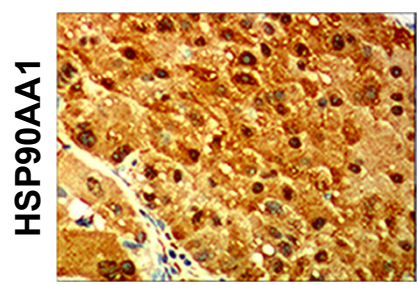

IOD: $423.1 \pm 24.4$

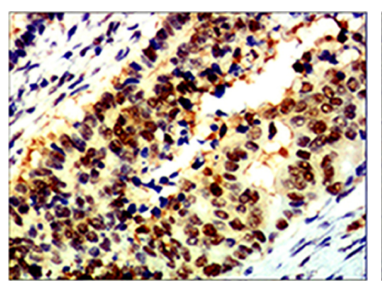

IOD: $258.6 \pm 20.7$

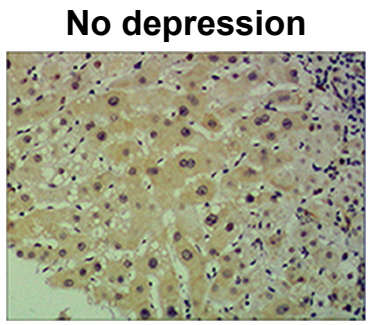

IOD: $168.6 \pm 21.8$

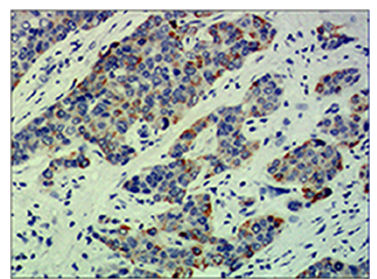

IOD: $193.9 \pm 11.9$

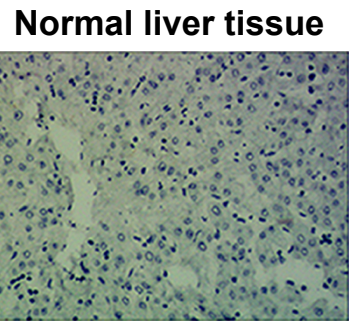

IOD: $68.6 \pm 8.5$

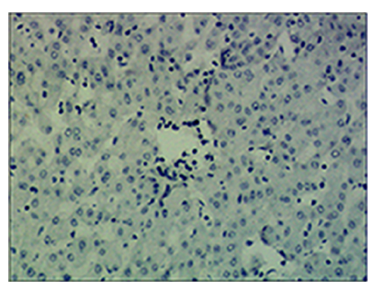

IOD: $60.2 \pm 10.4$

Figure 2 Levels of mRNAs encoding HSP90AAI or HSPA8 based on qRT-PCR (A). Levels of HSP90AAI and HSPA8 protein based on Western blotting (B). Levels of HSP90AAI and HSPA8 protein based on immunohistochemistry (magnification, 200 $\times$ ) (C).

Note: $* * P<0.01 ; * * * P<0.001$.

Abbreviation: qRT-PCR, quantitative reverse transcription polymerase chain reaction.

leading to the release of various growth factors and cytokines that promote the migration, proliferation, and survival of neonatal endothelial cells around the tumor. This leads to vascular proliferation around the tumor. VEGF-AKT drives a classic signaling pathway that promotes endothelial cell proliferation and survival, inhibits apoptosis, and promotes angiogenesis. Some studies have shown that VEGF-triggered cascades cause the downstream effector PI3K-AKT to inhibit 
A

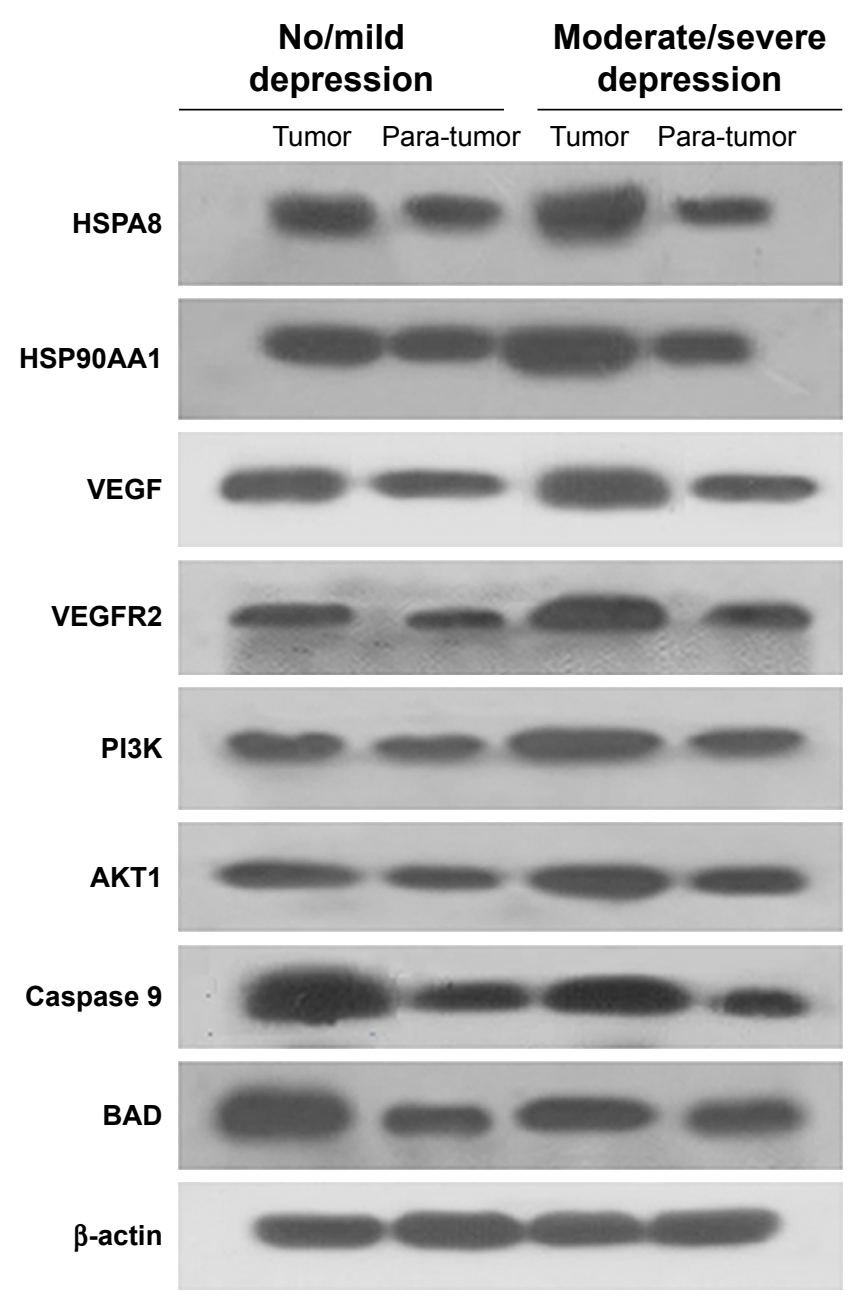

B
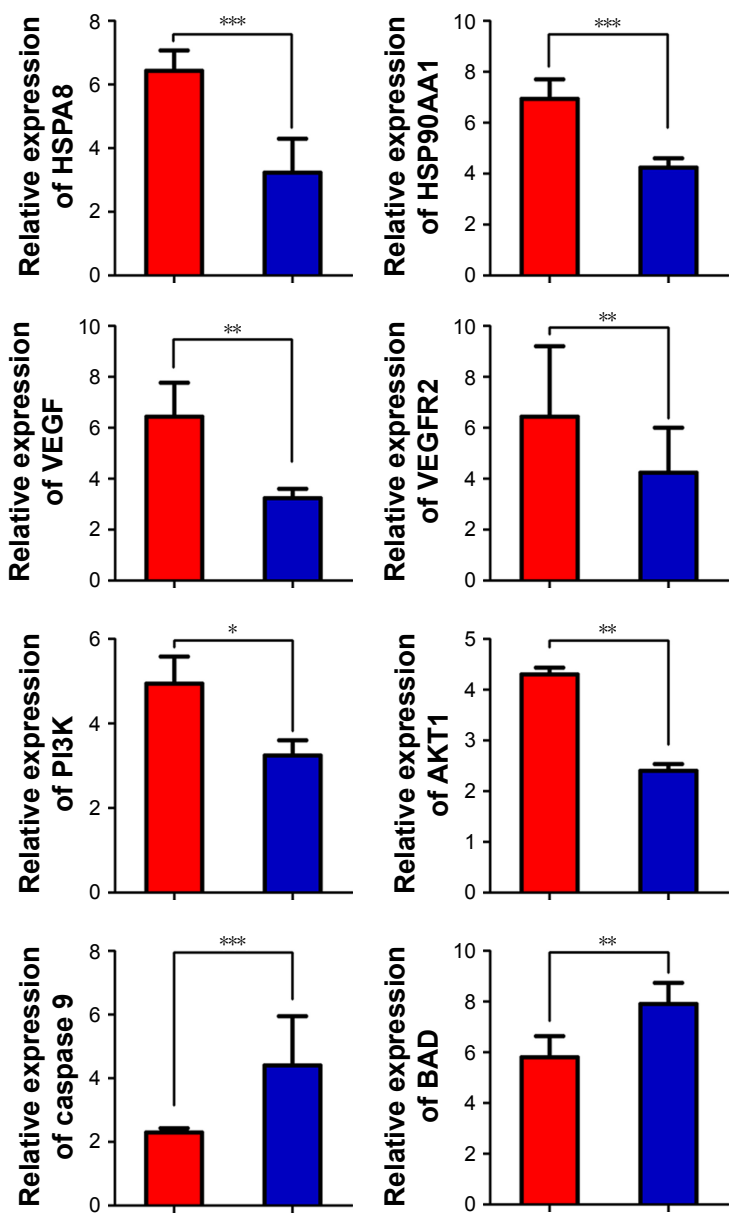

Moderate/severe depression

Mild/no depression

Figure 3 Comparison of expression of HSP90AAI, HSPA8, VEGF, VEGFR2, PI3K, AKTI, caspase 9, and BAD between HCC patients with moderate/severe or mild/no depression, based on Western blotting $(\mathbf{A})$ and $\mathrm{qRT}-\mathrm{PCR}(\mathbf{B})$.

Note: $* P<0.05 ; * * P<0.01$; *** $P<0.00$ I.

Abbreviations: HCC, hepatocellular carcinoma; $\mathrm{RRT}-\mathrm{PCR}$, quantitative reverse transcription polymerase chain reaction.

BAD and caspase 9, leading to reduced tumor cell apoptosis. The VEGF-AKT pathway can also promote endothelial cell proliferation and angiogenesis through mTORC2 and FOXO1. 19,37-39

Limitations of our study include the fact that the data come from a small patient population at a single center, which increases the risk of systematic errors and bias. Follow-up was relatively short; as a result, few patients underwent surgical resection after tumor recurrence during the study. It would be important to assay recurrent tumors for HSP90AA1/HSPA8 expression, which we plan to do in future work.

\section{Conclusion}

Our findings suggest that a substantial proportion of HCC patients in this HCC-endemic region of Guangxi suffer depression, and that moderate/severe depression can significantly affect post hepatectomy prognosis. Moderate/severe depression in HCC may be associated with upregulation of HSP90AA1 and HSPA8, which in turn correlates with activation of the VEGF/VEGFR2 pathway. This activation may contribute to HCC recurrence. Future studies should explore the potential usefulness of HSP90AA1 and HSPA8 as biomarkers of depression-related HCC, which may facilitate early diagnosis and individualized prevention and treatment. HCC patients who have moderate/severe depression and high expression of HSP90AA1 and HSPA8 may benefit from more intensive psychological intervention and postsurgical care as well as more frequent follow-up.

\section{Acknowledgments}

The authors are grateful to the Department of Hepatobiliary Surgery, Tumor Hospital Affiliated of Guangxi Medical 
University for the help with this paper. This work was supported by Traditional Chinese Medicine Science and Technology projects of the Ministry of Health of Guangxi Province (GZLC16-36), the Guangxi Medical Care Appropriate Technology Research and Development (S201417-03), the Guangxi University of Science and Technology Research Projects (KY2015LX056), and the Graduate Course Construction Project of Guangxi Medical University (YJSA2017014).

\section{Disclosure}

The authors report no conflicts of interest in this work.

\section{References}

1. Seeff LB, Hoofnagle JH. Epidemiology of hepatocellular carcinoma in areas of low hepatitis B and hepatitis C endemicity. Oncogene. 2006; 25(27):3771-3777.

2. Jemal A, Bray F, Center MM, Ferlay J, Ward E, Forman D. Global cancer statistics. CA Cancer J Clin. 2011;61(2):69-90.

3. European Association for the Study of the Liver. EASL 2017 Clinical Practice Guidelines on the management of hepatitis B virus infection. J Hepatol. 2017;67(2):370-398.

4. Reiche EM, Morimoto HK, Nunes SM. Stress and depression-induced immune dysfunction: implications for the development and progression of cancer. Int Rev Psychiatry. 2005;17(6):515-527.

5. Goddard AW, Ball SG, Martinez J, et al. Current perspectives of the roles of the central norepinephrine system in anxiety and depression. Depress Anxiety. 2010;27(4):339-350.

6. Donovan KA, Jacobsen PB. Fatigue, depression, and insomnia: evidence for a symptom cluster in cancer. Semin Oncol Nurs. 2007;23(2): 127-135.

7. Yamashita Y, Taketomi A, Shirabe K, et al. Outcomes of hepatic resection for huge hepatocellular carcinoma ( $\geq 10 \mathrm{~cm}$ in diameter). $J$ Surg Oncol. 2011;104(3):292-298.

8. Baltrusch HJ, Stangel W, Waltz ME. Cancer from the biobehavioral perspective: the type C pattern. Act Nerv Super (Praha). 1988;30(1): $18-21$.

9. Miyaki M, Iijma T, Iskii R, et al. Increased frequency of sporadic tumours from type C behavior. Jpn J Clin Oncol. 2002;2(5):101-115.

10. Fan SY, Eiser C, Ho MC. Health-related quality of life in patients with hepatocellular carcinoma: a systematic review. Clin Gastroenterol Hepatol. 2010;8(7):559-564.e1-e10.

11. Palmieri VO, Santovito D, Margari F, et al. Psychopathological profile and health-related quality of life (HRQOL) in patients with hepatocellular carcinoma (HCC) and cirrhosis. Clin Exp Med. 2015;15(1): 65-72.

12. Koh C, Zhao X, Samala N, Sakiani S, Liang TJ, Talwalkar JA. AASLD clinical practice guidelines: a critical review of scientific evidence and evolving recommendations. Hepatology. 2013;58(6):2142-2152.

13. Bruix J, Sherman M; American Association for the Study of Liver Diseases. Management of hepatocellular carcinoma: an update. Hepatology. 2011;53(3):1020-1022.

14. Martin G, Copeland J, Gates P, Gilmour S. The Severity of Dependence Scale (SDS) in an adolescent population of cannabis users: reliability, validity and diagnostic cut-off. Drug Alcohol Depend. 2006;83(1): 90-93.

15. Zigmond AS, Snaith RP. The hospital anxiety and depression scale. Acta Psychiatr Scand. 1983;67(6):361-370.

16. Xiang X, Qin HG, You XM, et al. Expression of P62 with hepatocellular carcinoma involving hepatitis B virus infection and aflatoxin B1 exposure. Cancer Med. 2017;6(10):2357-2369.
17. Xiang X, Zhong JH, Wang YY, et al. Distribution of tumor stage and initial treatment modality in patients with primary hepatocellular carcinoma. Clin Transl Oncol. 2017;19(7):891-897.

18. Zhong JH, Ke Y, Gong WF, et al. Hepatic resection associated with good survival for selected patients with intermediate and advanced-stage hepatocellular carcinoma. Ann Surg. 2014;260(2):329-340.

19. Graupera M, Guillermet-Guibert J, Foukas LC, et al. Angiogenesis selectively requires the p110alpha isoform of PI3K to control endothelial cell migration. Nature. 2008;453(7195):662-666.

20. Pandey P, Saleh A, Nakazawa A, et al. Negative regulation of cytochrome c-mediated oligomerization of Apaf-1 and activation of procaspase-9 by heat shock protein 90 . EMBO J. 2000;19(16):4310-4322.

21. Jego G, Hazoume A, Seigneuric R, Garrido C. Targeting heat shock proteins in cancer. Cancer Lett. 2013;332(2):275-285.

22. Kang GH, Lee EJ, Jang KT, et al. Expression of HSP90 in gastrointestinal stromal tumours and mesenchymal tumours. Histopathology. 2010;56(6):694-701.

23. Soroka J, Wandinger SK, Mausbacher N, et al. Conformational switching of the molecular chaperone Hsp90 via regulated phosphorylation. Mol Cell. 2012;45(4):517-528.

24. Li Y, Zhang T, Schwartz SJ, Sun D. Sulforaphane potentiates the efficacy of 17-allylamino 17-demethoxygeldanamycin against pancreatic cancer through enhanced abrogation of Hsp90 chaperone function. Nutr Cancer. 2011;63(7):1151-1159.

25. Li W, Sahu D, Tsen F. Secreted heat shock protein-90 (Hsp90) in wound healing and cancer. Biochim Biophys Acta. 2012;1823(3):730-741.

26. Nimmanapalli R, O’Bryan E, Bhalla K. Geldanamycin and its analogue 17-allylamino-17-demethoxygeldanamycin lowers Bcr-Abl levels and induces apoptosis and differentiation of Bcr-Abl-positive human leukemic blasts. Cancer Res. 2001;61(5):1799-1804.

27. Blagosklonny MV, Toretsky J, Bohen S, Neckers L. Mutant conformation of p53 translated in vitro or in vivo requires functional HSP90. Proc Natl Acad Sci U S A. 1996;93(16):8379-8383.

28. Shiono Y, Fujita Y, Oka S, Yamazaki Y. ATPase inhibitors suppress actinomycin D-induced apoptosis in leukemia cells. Anticancer Res. 2002;22(5):2907-2911.

29. Zhao C, Wang EH. Heat shock protein 90 suppresses tumor necrosis factor $\alpha$ induced apoptosis by preventing the cleavage of Bid in NIH3T3 fibroblasts. Cell Signal. 2004;16(3):313-321.

30. Udono H, Srivastava PK. Heat shock protein 70-associated peptides elicit specific cancer immunity. $J$ Exp Med. 1993;178(4):1391-1396.

31. Lee TK, Han JS, Fan ST, et al. Gene delivery using a receptor-mediated gene transfer system targeted to hepatocellular carcinoma cells. Int $J$ Cancer. 2001;93(3):393-400.

32. Gross C, Hansch D, Gastpar R, Multhoff G. Interaction of heat shock protein 70 peptide with NK cells involves the NK receptor CD94. Biol Chem. 2003;384(2):267-279.

33. Chuma M, Sakamoto M, Yamazaki K, et al. Expression profiling in multistage hepatocarcinogenesis: identification of HSP70 as a molecular marker of early hepatocellular carcinoma. Hepatology. 2003; 37(1):198-207.

34. Frumovitz M, Sood AK. Vascular endothelial growth factor (VEGF) pathway as therapeutic target in gynecologic malignancies. Gynecol Oncol. 2007;104(3):768-778.

35. Ferrara NL. Vascular endothelial growth factor as a target for anticancer therapy. Oncologist. 2004;9(Suppl 1):2-10.

36. Semela D, Dufour JF. Angiogenesis and hepatocellular carcinoma. J Hepatol. 2004;41(5):864-880.

37. Sarbassov DD, Guertin DA, Ali SM, Sabatini DM, et al. Phosphorylation and regulation of Akt/PKB by the rictor-mTOR complex. Science. 2005;307(5712):1098-10101.

38. Jacinto E, Facchinetti V, Liu D, et al. SIN1/MIP1 maintains rictormTOR complex integrity and regulates Akt phosphorylation and substrate specificity. Cell. 2006;127(1):125-137.

39. Rotllan N, Wanschel AC, Fernández-Hernando A, et al. Genetic evidence supports a major role for Akt1 in VSMCs during atherogenesis. Circ Res. 2015;116(11):1744-1752. 


\section{Publish your work in this journal}

OncoTargets and Therapy is an international, peer-reviewed, open access journal focusing on the pathological basis of all cancers, potential targets for therapy and treatment protocols employed to improve the management of cancer patients. The journal also focuses on the impact of management programs and new therapeutic agents and protocols on
Dovepress

patient perspectives such as quality of life, adherence and satisfaction. The manuscript management system is completely online and includes a very quick and fair peer-review system, which is all easy to use. Visit http://www.dovepress.com/testimonials.php to read real quotes from published authors.

\footnotetext{
Submit your manuscript here: http://www.dovepress.com/oncotargets-and-therapy-journal
} 